\title{
Non-surgical management of an oro-antral fistula in a patient with HIV infection
}

\author{
RM Logan, ${ }^{*}+$ EA Coates*
}

\begin{abstract}
Background: The risk of post-extraction complications is higher in patients who are immunosuppressed compared to other patients with normal immune function. In addition, invasive dental procedures are more likely to have serious complications in these patients. This case report demonstrates an effective non-surgical procedure to treat an oro-antral fistula in an HIV-infected man.

Methods: The oro-antral fistula was deepithelialized under local anaesthesia and the patient wore a surgical splint continuously, removing it only for cleaning, for an eight week period. Chlorhexidine gel was regularly applied to the fitting surface of the splint and the oro-antral communication. The patient was reviewed on a regular basis.

Results: This procedure resulted in resolution of the patient's symptoms within two weeks. Complete healing of the oro-antral fistula was evident following eight weeks of wearing the surgical splint. Conclusions: This procedure provided an effective method of treating an oro-antral fistula in an immunocompromised patient without causing any detrimental effects to the patient's overall health. Adequate pre-surgical assessment of patients prior to extractions is important in all patients to help prevent the occurrence of such complications.
\end{abstract}

Key words: Oro-antral fistula, HIV infection, AIDS, immunosuppression.

(Accepted for publication 24 April 2003.)

\section{INTRODUCTION}

The development of oro-antral fistulae or communications are not common events following the extraction of teeth. ${ }^{1,2}$ When they do occur they usually result as a consequence of extraction of teeth posterior to the maxillary canines. ${ }^{2,3}$ In particular the upper second molar followed by the upper first molar teeth are the most likely teeth to result in an oro-antral communication following their extraction. ${ }^{4}$

\footnotetext{
"Medically Compromised Patient Unit, Adelaide Dental Hospital.
} †Oral Pathology, Dental School, The University of Adelaide.
Following the creation of an oro-antral communication, the patient may experience various symptoms including air and fluids passing into the nose and mouth. The diameter of the communication usually decreases over time but often the 'tract' from the antrum to the mouth fails to heal and becomes lined by epithelium. Once this occurs the communication is referred to as a fistula. This fistula acts as a pathway for infection and can result in the development of acute sinusitis which further impedes healing.

The likelihood of the development of a communication is increased if there is pre-existing sinus disease, periapical infections, or if the floor of the sinus is in close proximity to the tooth roots (e.g., antrum floor dipping down between the roots of teeth). In addition, attempts to remove apices of teeth that have fractured during extraction may result in the creation of a communication between the maxillary sinus and oral cavity.

In the setting of human immunodeficiency virus (HIV) infection there have been very few reports of oroantral fistulae occurring following extraction of teeth. One case report described the spontaneous development of an oro-antral fistula in a patient with HIV infection in association with HIV-related periodontal disease. ${ }^{5}$ However, sinus disease is commonly encountered in patients with HIV infection. ${ }^{6}$ Chronic HIV-related sinusitis is often related to Pseudomonas aeruginosa, Staphylococcus aureus or anaerobic bacteria. ${ }^{6}$ Because of the increased prevalence of chronic sinusitis in these patients, the risk of the development of oro-antral communications is increased and when a communication occurs, either spontaneously or through a surgical event, healing is impeded by the presence of sinusitis.

The treatment of oro-antral fistulae usually involves surgical intervention. Various techniques have been reported in the literature including local flaps (e.g., palatal rotational advancement and buccal advancement flaps), distant flaps (e.g., tongue flaps) or grafts (e.g., bone). ${ }^{1,7}$ However, as previously mentioned if the fistulae are small (i.e., less than $5 \mathrm{~mm}$ ) and sinus disease is absent or eliminated, then spontaneous healing may occur. It is also important that the 


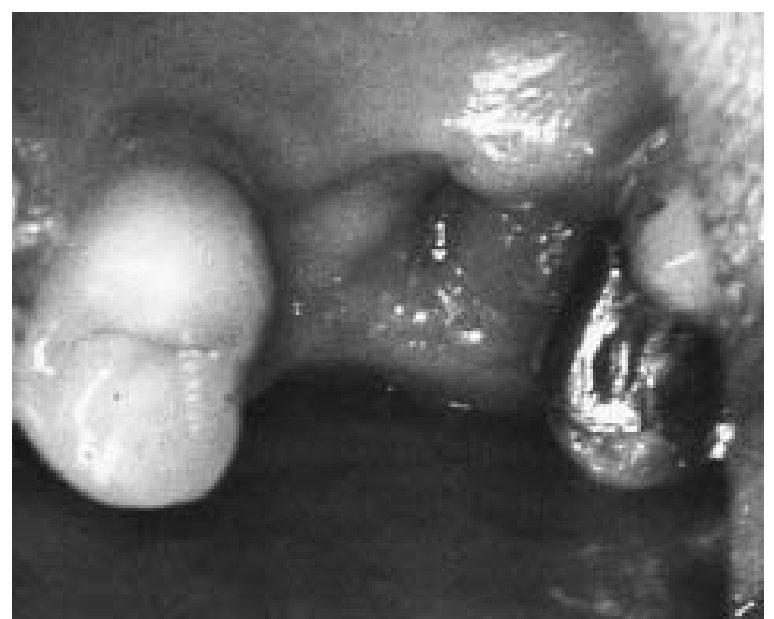

Fig 1. An oro-antral fistula present at the site of the upper left first molar.

epithelial lining of the fistula is removed in order to facilitate healing. ${ }^{3}$

The purpose of this case report is to describe an alternative non-surgical treatment for an oro-antral fistula in a patient with HIV infection.

\section{CASE REPORT}

A 34-year-old man who was infected with HIV was referred to the Medically Compromised Patient Unit of the Adelaide Dental Hospital for the management of an oro-antral fistula that developed following extraction of his upper left first molar three months previously. In terms of his HIV disease he was significantly immunosuppressed (CD4 T+ lymphocyte count 82 cells $/ \mathrm{mL}$ ). Prior to having the tooth extracted, the patient had not had any problems with dental treatment.

Specialist surgical opinion favoured hospitalization of the patient and surgical closure of the fistula. However, the patient's general medical practitioner was concerned about the risks involved in such procedures because of the patient's significant immunosuppression and requested a second specialist opinion from the Medically Compromised Patient Unit of the Adelaide Dental Hospital.

On initial examination, a small oro-antral fistula was present at the site of the upper left first molar (Fig 1).

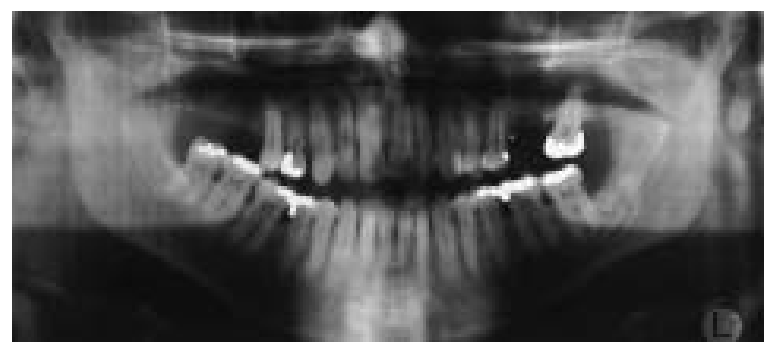

Fig 2. Orthopantomograph of the dentition at the time of the initial examination illustrating the state of the remaining alveolus. A small fragment of amalgam is noted adjacent to the site of extraction.

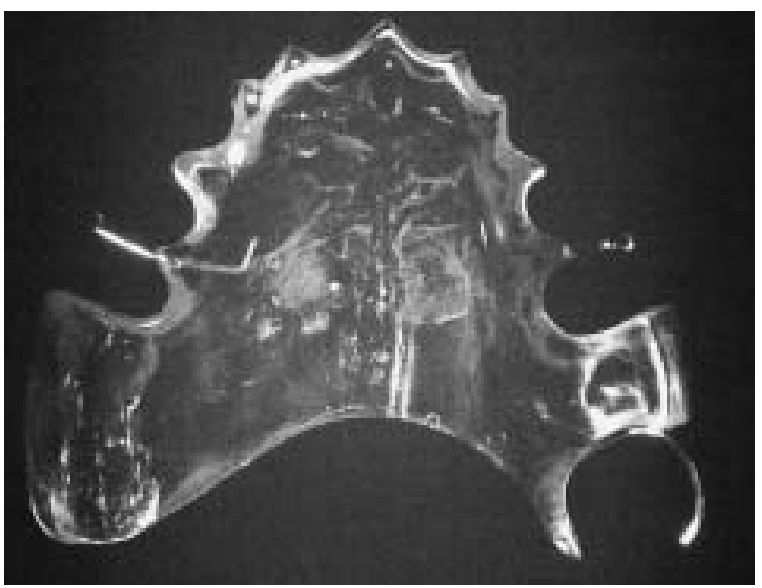

Fig 3. Acrylic surgical splint constructed to cover the oro-antral fistula.

The patient complained that his main problem was not pain or discomfort, but that drinking and eating was difficult as fluids escaped into his nasal cavity. The orthopantomogram radiograph taken at the time of the initial appointment demonstrated the close proximity of the roots of the upper posterior teeth to the maxillary antrum as well as the significant loss of the remaining alveolus in the area of the extraction of the 26 (Fig 2). The patient had no other oral problems apart from moderate xerostomia that was attributed to his HIV infection.

It was felt that surgical intervention was contraindicated as a first line treatment in this patient because of his significant immunosuppression. In addition it was thought that because the fistula was not large and that there was no obvious sinus infection present, simple local measures would facilitate healing.

Models of the upper and lower teeth were made and these were used to construct an acrylic surgical splint (Fig 3) that entirely covered the edentulous area and the fistula. The splint was adjusted so that it was as comfortable as possible when worn for extended periods of time.

The epithelial lining of the oro-antral fistula was removed by carefully curetting the lesion under local anaesthesia. The fitting surface of the splint was coated with chlorhexidine gel $(0.02$ per cent) prior to insertion. The patient was instructed to wear the splint continually for the next two weeks removing it only for cleaning after meals at which time he was to apply more chlorhexidine gel to the fitting surface in the area of the fistula. He was also advised to avoid or minimize any activity that increased intra-oral or intranasal pressure (e.g., smoking, drinking through straws, blowing his nose etc). A review appointment was arranged two weeks later.

\section{RESULTS}

On review, the intra-oral defect had reduced in size and there was a marked improvement in the patient's symptoms. At this stage in the patient's management 


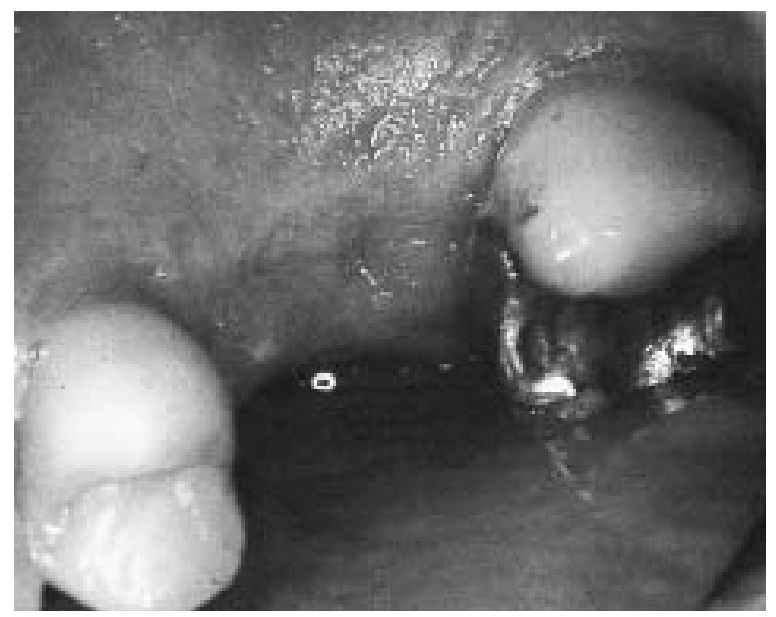

Fig 4. Clinical appearance of the site of the upper left first molar eight weeks after the treatment started.

the only complicating factor was mild pseudomembranous candidiasis. It is likely that the use of the splint in conjunction with xerostomia and immunosuppression resulted in the development of the candidiasis. It was effectively treated using Daktarin oral gel (miconazole $20 \mathrm{mg} / \mathrm{mL}$ ). Because of the improvement in symptoms and the oral candidiasis, it was decided to reduce the amount of time that the patient wore the splint.

At eight weeks after the initiation of treatment, the defect had healed and symptoms completely resolved (Fig 4). At 12 months following initial presentation, the patient remained symptom free and the clinical appearance of the area is demonstrated in Fig 5.

\section{DISCUSSION}

There are very few publications regarding the risks of patients with HIV infection undergoing invasive dental procedures. ${ }^{8}$ According to Dodson et al. ${ }^{9}$ the risk of post-extraction complications in patients with HIV

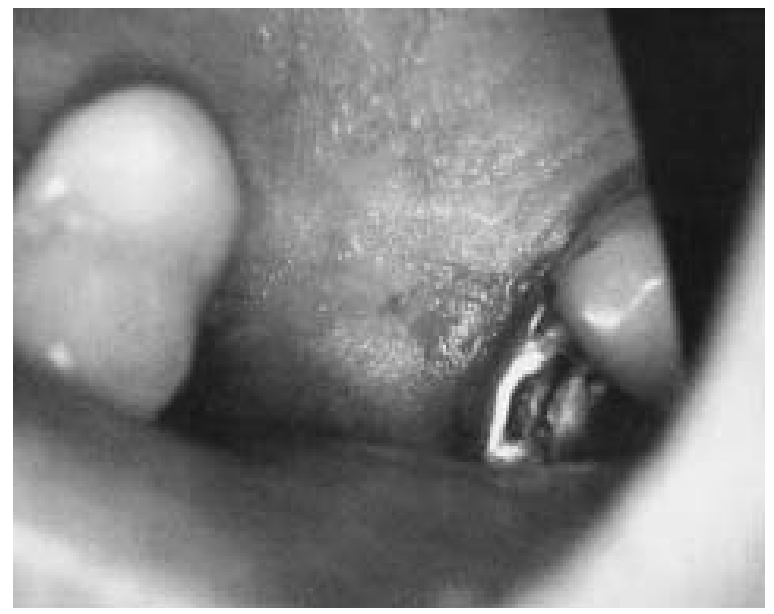

Fig 5. Clinical appearance of the site of the oro-antral fistula 12 months after the initial presentation of the patient to the clinic showing complete closure of the communication. infection increases with the level of immunosuppression and the rate of post-extraction complications in the setting of HIV has been reported to range between 0.9-22 per cent. ${ }^{10,11}$

The most frequent post-extraction complications in patients with HIV infection include delayed healing, alveolitis and surgical wound infection..$^{12}$ There are few reports in the literature of oro-antral fistulae occurring in association with HIV infection. Felix et al. ${ }^{5}$ reported on an oro-antral fistula that occurred as an unusual complication of necrotizing ulcerative stomatitis rather than following a dental extraction. Surgical closure of oro-antral fistulae involving local flap procedures may have some degree of failure. ${ }^{13}$ The management of a patient with a large chronic oro-antral fistula that had failed to be treated by several local flap procedures was reported by Godfrey. ${ }^{13}$ In that case report ultimate resolution of the problem was achieved using vascularized temporoparietal fascia.

In the present case report, the patient's general medical practitioner was reticent to allow the patient to undergo surgical treatment because of the degree of immunosuppression. Surgical closure of the lesion would have taken an extended length of time, required general anaesthesia and hospitalization of the patient. It has been reported that small fistulae, $5 \mathrm{~mm}$ or less in diameter are likely to heal spontaneously. However, if they fail to heal or if they are larger, then surgical management is indicated. ${ }^{1}$ In the present case it was felt that the fistula was small and that given improvement in local measures (i.e., de-epithelialization as well as the use of the splint) healing should be facilitated. Additionally our patient did not have any clinical signs or symptoms of sinus infection. However, we did prescribe a course of broad spectrum antibiotics as the use of antibiotics is recommended in the literature even in the absence of sinus disease as a prophylactic measure during treatment. ${ }^{3}$ Compared to surgical management, the procedure that we have described was minimally invasive and appeared to have very few risks associated with it.

Adequate pre-surgical assessment of all patients prior to extractions is important in order to help prevent the occurrence of such complications. This includes evaluation of both general and local factors that may influence healing such as systemic health (e.g., immunosuppression) and factors such as pre-existing sinus disease or periapical infection. Where there is a possibility of antral perforation either by anatomical variation or from the presence of chronic infection, the use of a splint placed immediately following extraction of teeth may well prevent the development of a fistula. In such situations wearing the splint would be necessary for a much shorter period of time (e.g., one to two weeks).

\section{CONCLUSION}

This case report describes a procedure that provided a simple and effective method of treating an oro-antral fistula in an immunocompromised patient without 
causing any detrimental effects to the patient's overall health.

\section{REFERENCES}

1. Awang MN. Closure of oroantral fistula. Int J Oral Maxillofac Surg 1988;17:110-115.

2. Punwutikorn J, Waikakul A, Pairuchvej V. Clinically significant oroantral communications - a study of incidence and site. Int J Oral Maxillofac Surg 1994;23:19-21.

3. Moore JR, Gillbe GV. Principles of oral surgery. 4th edn. Manchester University Press, 1991.

4. Guven O. A clinical study on oroantral fistulae. J Craniomaxillofac Surg 1998;26:267-271.

5. Felix DH, Wray D, Smith GL, Jones GA. Oro-antral fistula: an unusual complication of $\mathrm{HIV}$-associated periodontal disease. $\mathrm{Br}$ Dent J 1991;171:61-62.

6. Tami TA. The management of sinusitis in patients infected with the human immunodeficiency virus (HIV). Ear Nose Throat J 1995;74:360-363.

7. el-Hakim IE, el-Fakharany AM. The use of the pedicled buccal fat pad (BFP) and palatal rotating flaps in closure of oroantral communications and palatal defects. J Laryngol Otol 1999;113:834-838.

8. Patton LL, Shugars DA, Bonito AJ. A systematic review of complication risks for HIV-positive patients undergoing invasive dental procedures. J Am Dent Assoc 2002;133:195-203.
9. Dodson TB, Perrett DH, Gongloff RK, Kaban LB. Human immunodeficiency virus serostatus and the risk of postextraction complications. Int J Oral Maxillofac Surg 1994;23:100-103.

10. Glick M, Abel SN, Muzyka BC, DeLorenzo M. Dental complications after treating patients with AIDS. J Am Dent Assoc 1994;125:296-301.

11. Dodson TB. HIV status and the risk of post-extraction complications. J Dent Res 1997;76:1644-1652.

12. Diz Dios P, Fernandez Feijoo J, Vazquez Garcia E. Tooth extraction in HIV sero-positive patients. Int Dent J 1999;49:317321.

13. Godfrey PM. Sinus obliteration for chronic oro-antral fistula: a case report. Br J Plast Surg 1993;46:341-342.

Address for correspondencelreprints:

Dr Richard M Logan

Oral Pathology Unit

Dental School

Faculty of Health Science

The University of Adelaide

North Terrace

Adelaide, South Australia 5005

Email: richard.logan@adelaide.edu.au 